Check for updates

Cite this: Analyst, 2018, 143, 493

Received 8th November 2017, Accepted 19th December 2017

DOI: $10.1039 / c 7 a n 01823 a$

rsc.li/analyst

\section{Cavity enhanced liquid-phase stopped-flow kinetics}

\author{
Zuzana Bajuszova, Hanan Naif, Zulfiqur Ali, Joseph McGinnis and Meez Islam (D) *
}

The first application of liquid-phase broadband cavity enhanced spectroscopy (BBCEAS) to the measurement of stopped-flow kinetics is reported. The stopped-flow technique is widely used for the study of the kinetics of fast liquid-phase reactions down to millisecond timescales. UV-visible absorption spectroscopy is commonly used as the detection method. Increased sensitivity can potentially allow reactions which are too fast to be measured, to be studied by slowing down the reaction rate through the use of lower concentration of reactants. A simple low cost BBCEAS experimental setup was coupled to a commercial stopped-flow instrument. Comparative standard absorption measurements were also made using a UVvisible double-beam spectrometer as the detector. Measurements were made on the reaction of potassium ferricyanide with sodium ascorbate under pseudo-first order conditions at pH 8 and pH 9.2 A cavity enhancement factor (CEF) of 78 at $434 \mathrm{~nm}$ was obtained whilst the minimum detectable change in the absorption coefficient $\alpha_{\min }(t)$, was $1.35 \times 10^{-5} \mathrm{~cm}^{-1} \mathrm{~Hz}^{-1 / 2}$. The kinetic data at $\mathrm{pH} 9.2$ was too fast to be measured using conventional spectroscopy, whilst the BBCEAS measurements allowed 30 fold lower concentration of reactants to be used which slowed down the reaction rate enough to allow the rate constant to be determined. The BBCEAS results showed a 58 fold improvement in sensitivity over the conventional measurements and also compared favourably with the relatively few previous liquid-phase cavity enhanced kinetic studies which have been performed using significantly more complex and expensive experimental setups.

\section{Introduction}

The measurement of the kinetics of chemical reactions is recognised as being of fundamental importance and finds a large number of applications ranging from investigations of enzyme kinetics, protein binding, and atmospheric chemistry, to microorganism growth. ${ }^{1,2}$ For the measurement of liquidphase chemical kinetics on time scales of a few seconds down to a few milliseconds, the stopped-flow method in conjunction with absorbance based spectrometers is commonly used. This relies on the rapid mixing of small volumes of reactants which is quickly stopped. However, stopped-flow systems typically have a "dead time", the time required to mix the reagents and begin data collection, of 2-10 ms. If the half-life of a reaction is much less than this, the reaction will be essentially complete before any data can be obtained. Lowering the concentrations of reagents could be used to slow reactions down and would have the added advantage of reducing the cost of potentially expensive consumables. ${ }^{3}$ The stopped-flow method is also used in conjunction with other techniques for the spectrophotometric determination of analytes ${ }^{4,5}$ and an increase in

School of Science and Engineering, Teesside University, Borough Road, Middlesbrough, TS1 3BA, UK. E-mail: m.islam@tees.ac.uk sensitivity would allow lower concentration of analytes to be detected.

The sensitivity of absorption spectroscopy has been greatly enhanced recently by the use of optical cavity based techniques. These approaches provide a substantial improvement over conventional absorption spectroscopy by increasing the effective path length of measurement and thus the sensitivity of the measurement. The first technique was called cavity ringdown spectroscopy (CRDS) and was based on the measurement of the decay time of the intensity of light exiting the cavity. ${ }^{6}$ A decade later an experimentally simpler variant based on the measurement of the integrated intensity output from the cavity was proposed and is most commonly known as cavity enhanced absorption spectroscopy (CEAS). ${ }^{7,8}$ Further development and simplification of CEAS have included the use of cheap broadband light sources such as high intensity light emitting diodes (LED) instead of laser light sources leading to the technique broadband CEAS (BBCEAS). ${ }^{9}$ To date, the vast majority of cavity based studies have been performed on gasphase analytes as the background scattering and absorption losses are much lower than for the condensed phases. However, over the past few years, several studies have been reported on liquid-phase species despite the greater scattering and absorption losses as many important analytes are found in the liquid-phase. The first liquid-phase cavity study using a 
LED light source was reported by Islam et al., $2007^{10}$ on measurements in a $2 \mathrm{~mm}$ cuvette, followed by a study in a $20 \mathrm{~cm}$ cell in which the analytes were in direct contact with the cavity mirrors. ${ }^{11}$ The group also successfully demonstrated BBCEAS as a detector for high performance liquid chromatography (HPLC), one of the most widely used techniques in analytical chemistry. ${ }^{12}$ Recent developments in this field include the application of BBCEAS to microfluidic devices, ${ }^{13}$ sensitive liquid-phase measurements employing a low cost prism based webcam spectrometer as the detector ${ }^{14}$ and measurements in a microtitre plate using BBCEAS. ${ }^{15}$ To date there have been relatively few liquid-phase kinetic studies which have used cavity enhanced techniques. ${ }^{16-20}$ These have generally used bespoke, complex and expensive experimental setups.

We demonstrate in this study the first application of a cavity enhanced technique to a commercial stopped-flow kinetics instrument. The technique chosen was BBCEAS due to its simplicity and low cost. The reaction chosen for study was that between potassium ferricyanide and sodium ascorbate, as it has been studied previously using stopped-flow apparatus. $^{21}$ The reaction rate is also $\mathrm{pH}$ dependent which meant that the reaction rate could be varied over a wide range by changing the $\mathrm{pH}$. Thus it was suitable for testing the performance of the cavity enhanced stopped-flow instrument against measurement using conventional UV-visible absorption spectroscopy. ${ }^{22}$

\section{Experimental section}

\section{The cavity enhanced stopped-flow apparatus}

A schematic of the apparatus is shown in Fig. 1. The light source used was a $3 \mathrm{~W}$ Luxeon Star white LED (Philips Lumileds Lighting Company) which emitted over the wavelength range 420-700 $\mathrm{nm}$. The light output was coupled by a $1000 \mu \mathrm{m}$ diameter fibre optic into an optical cavity formed by two concave, high reflectivity dielectric mirrors $(R \geq 0.99$ between $420 \mathrm{~nm}$ and $640 \mathrm{~nm}$, radius of curvature $=-100 \mathrm{~mm}$, Layertec, Germany) separated by a distance of $10 \mathrm{~cm}$. Light exiting the cavity was focussed onto the entrance slit of a compact CCD spectrometer (AvaSpec-ULS2048 StarLine). A commercial stopped-flow apparatus (SFA-20 Rapid Kinetics Spectrometer Accessory, TgK Scientific, UK) was used. It was coupled to a thermostatted water bath for temperature control. The reaction studied was that between sodium ascorbate which forms the ascorbate ion $\left[\mathrm{HA}^{-}\right]$in solution and potassium ferricyanide, $\mathrm{K}_{3}\left[\mathrm{Fe}(\mathrm{CN})_{6}\right]$ which forms the ferricyanide ion in solution (Sigma Aldrich, UK). The reaction was followed by monitoring the absorbance of ferricyanide.

$$
\mathrm{HA}^{-}+2\left[\mathrm{Fe}(\mathrm{CN})_{6}\right]^{3-} \rightarrow 2\left[\mathrm{Fe}(\mathrm{CN})_{6}\right]^{4-}+\mathrm{A}+\mathrm{H}^{+}
$$

The flow-cell was held in an adjustable kinematic mount and was positioned equidistant between the mirrors. The $2 \mathrm{~mm}$ path length side of the flow-cell was used for all

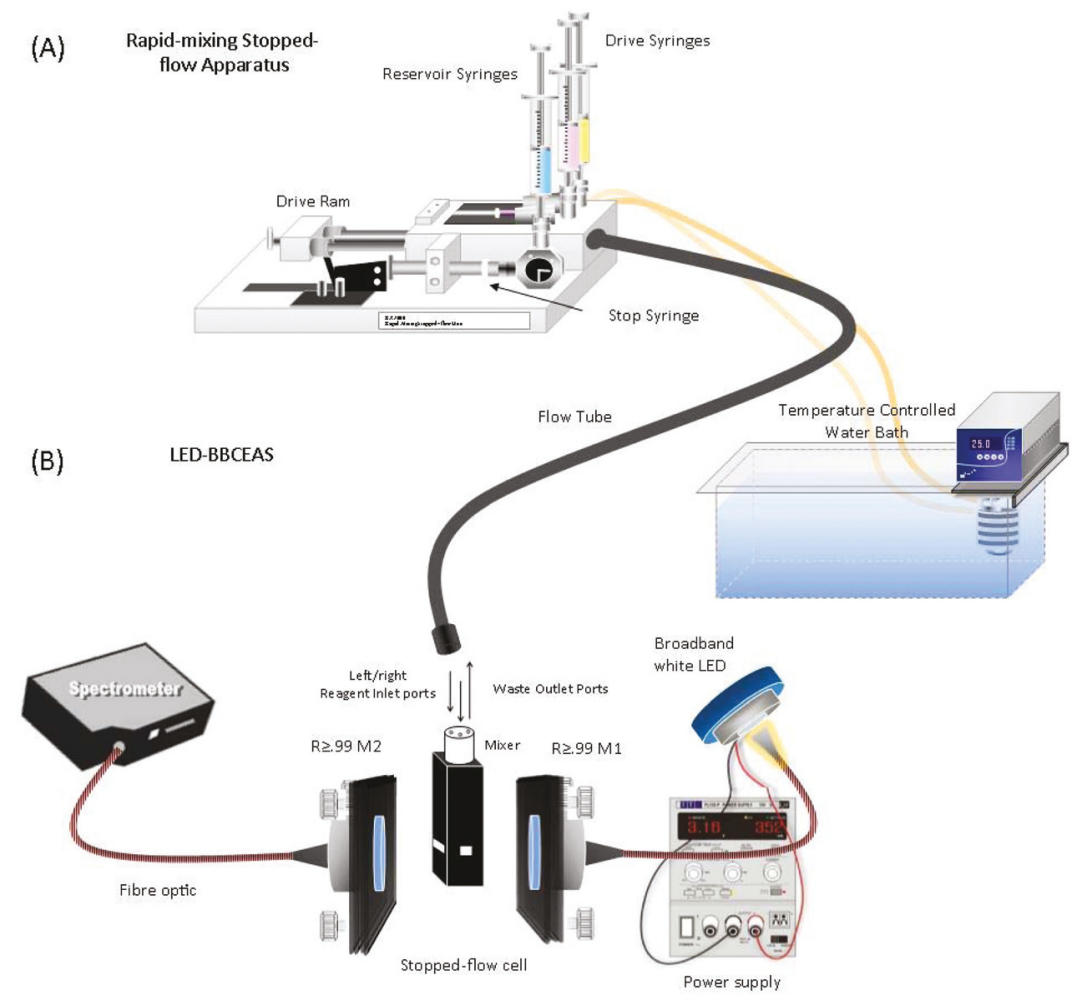

Fig. 1 A schematic of the cavity enhanced stopped-flow setup. (A) Thermostat controlled SFA-20 Rapid Kinetics accessory and flow-cell (B) BBCEAS setup with $R \geq 0.99$ cavity mirror set, LED light source and CCD spectrograph. 
measurements as the $10 \mathrm{~mm}$ path length side had surface scratches which led to poor light transmission. The water bath was set to a constant temperature of $25{ }^{\circ} \mathrm{C}$ for all measurements. To simplify the kinetic analysis, the reaction was performed under pseudo-first order conditions for ferricyanide with the concentration of sodium ascorbate in large excess of at least 10 fold greater. To determine the second order rate constant $\left(k_{2}\right)$ for the overall reaction, experiments were conducted by adding varied concentrations of sodium ascorbate to $0.01 \mathrm{mM}$ ferricyanide. The rate of reaction has been found to increase with $\mathrm{pH}$. The first set of measurements were made at $\mathrm{pH} 8$, followed by a set of measurements at $\mathrm{pH} 9.2$ to increase the reaction rate. Deionised water was used as a reference solution. Comparative conventional measurements were made by placing the flow-cell into the sample compartment of a Jasco V-630 double beam UV-visible spectrometer.

\section{Experimental methodology}

The absorption of the analyte in the cavity was measured by first recording a reference spectrum with the pure solvent $\left(I_{\mathrm{o}}\right)$ and then the sample spectrum (I) containing different concentrations of the analyte. Previous studies have shown that for cavity experiments the cavity absorption can be expressed as; ${ }^{23}$

$$
\left(\frac{I_{0}(\lambda)}{I(\lambda)}-1\right)_{\text {cavity }}=\frac{2.303 \varepsilon_{\lambda} C l}{(1-R(\lambda))}
$$

where, $l$ is the base path length through the sample in the cavity in $\mathrm{cm}, R(\lambda)$ is the average wavelength dependent mirror reflectivity, $\varepsilon_{\lambda}$ is the wavelength dependent extinction coefficient $\left(\mathrm{M}^{-1} \mathrm{~cm}^{-1}\right)$, and $C$ is the concentration of the sample (M). The term $\varepsilon_{\lambda} C l$ is equivalent to the single pass absorbance $\left(\log _{10} I_{\mathrm{o}} / I\right)$. This expression, which is valid for all reasonable absorptions, shows that in a cavity experiment the cavity absorption, $\left(\frac{I_{0}(\lambda)}{I(\lambda)}-1\right)_{\text {cavity }}$ is linearly proportional to the concentration of the analyte. This is different from a single pass experiment where the absorbance $\left(\log _{10} I_{\mathrm{o}} / I\right)$ is linearly proportional to concentration. It can be seen from eqn (1) that the enhancement produced by a cavity experiment over a single pass experiment is due to the term $\frac{1}{(1-R(\lambda))}$. If this is set to be the wavelength dependent cavity enhancement factor (CEF), then the expression can be rearranged in terms of CEF;

$$
\operatorname{CEF}(\lambda)=\frac{\left(\frac{I_{\mathrm{o}}(\lambda)}{I(\lambda)}-1\right)_{\text {cavity }}}{2.303 \varepsilon_{\lambda} C l}
$$

If the cavity absorption is plotted against concentration and the gradient of the linear plot divided by $2.303 \varepsilon_{\lambda} l$, then a value of the CEF at a given wavelength and averaged over a range of concentrations is obtained. A linear regression is fitted and the gradient obtained by a least squares method. The standard deviation in the measurement of the gradient can also be used to calculate the standard deviation in the CEF value. The CEF value can be used to calculate the effective path length of measurement at a given wavelength $\left(l_{\mathrm{eff}}=l \times \mathrm{CEF}\right)$ where $l$ is the base path length. The effective path length is used in the calculation of the sensitivity of the measurement which is defined in terms of the minimum detectable change in the absorption coefficient $\alpha_{\text {min }}$ (also known as noise-equivalent absorption coefficient $\left.{ }^{24}\right)$. The time normalized $\alpha_{\min }(t)$ value with units of $\mathrm{cm}^{-1} \mathrm{~Hz}^{-1 / 2}$ for each measurement can be expressed as:

$$
\alpha_{\min }(t)=\frac{2.303 \Delta \mathrm{ABS}_{\min }(t)}{l_{\mathrm{eff}}}
$$

where $\Delta \mathrm{ABS}_{\min }(t)$ is the minimum detectable absorbance change in the cavity absorption spectrum normalized to one second of measurement by taking the total acquisition time in seconds $(\tau)$ into account by using the following expression: ${ }^{15}$

$$
\Delta \mathrm{ABS}_{\min }(t)=\Delta \mathrm{ABS}_{\min } \times \sqrt{\tau}
$$

This allows a fairer comparison of the sensitivity of an experiment.

The value of $\alpha_{\min }(t)$ could also be used to calculate the limit of detection (LOD) of an analyte which is defined as the smallest amount that can be reliably measured. ${ }^{10}$ It was calculated from the expression:

$$
\mathrm{LOD}=\frac{3 \alpha_{\min }(t)}{2.303 \varepsilon_{\lambda}}
$$

The order of the reaction with respect to each reagent was determined from the plot of the natural logarithm (ln) of absorbance versus time for ferricyanide and the plot of pseudofirst order rate constants $\left(k_{\mathrm{obs}}\right)$ versus $\left[\mathrm{HA}^{-}\right]$for ascorbate.

\section{Results}

\section{Measurement of the CEF and other figures of merit}

The absorption versus wavelength spectrum of ferricyanide was recorded over a $70 \mathrm{~nm}$ wavelength range through the $2 \mathrm{~mm}$ side of the flow-cell at a total acquisition time of $15 \mathrm{~ms}$ and is shown in Fig. 2. The inset shows the calibration plot in the concentration range $0.01 \mathrm{mM}$ to $0.07 \mathrm{mM}$ at a wavelength of $434 \mathrm{~nm}$. The graph also shows the gradient obtained from the slope of the fit. The selected wavelength of measurement at $434 \mathrm{~nm}$ was a compromise between the absorbance maximum of ferricyanide at $\sim 420 \mathrm{~nm}$, and the intensity output of the white LED which decreased sharply from $440 \mathrm{~nm}$ to $420 \mathrm{~nm}$. A separate single pass calibration was recorded over a higher concentration range of $0.1 \mathrm{mM}-0.9 \mathrm{mM}$ to obtain the molar extinction coefficient of ferricyanide at $434 \mathrm{~nm}$. This produced a value of $739.8 \mathrm{M}^{-1} \mathrm{~cm}^{-1}$. The results were used to calculate the average number of passes or CEF and also the $\alpha_{\min }$ and $\Delta \mathrm{ABS}_{\min }(t)$ values which are summarized in Table 1 .

\section{Measurements at $\mathbf{p H} 8$}

Kinetic traces. To investigate the suitability of BBCEAS as a technique to follow rapid reaction kinetics, measurements were made on the reaction between ferricyanide and sodium 

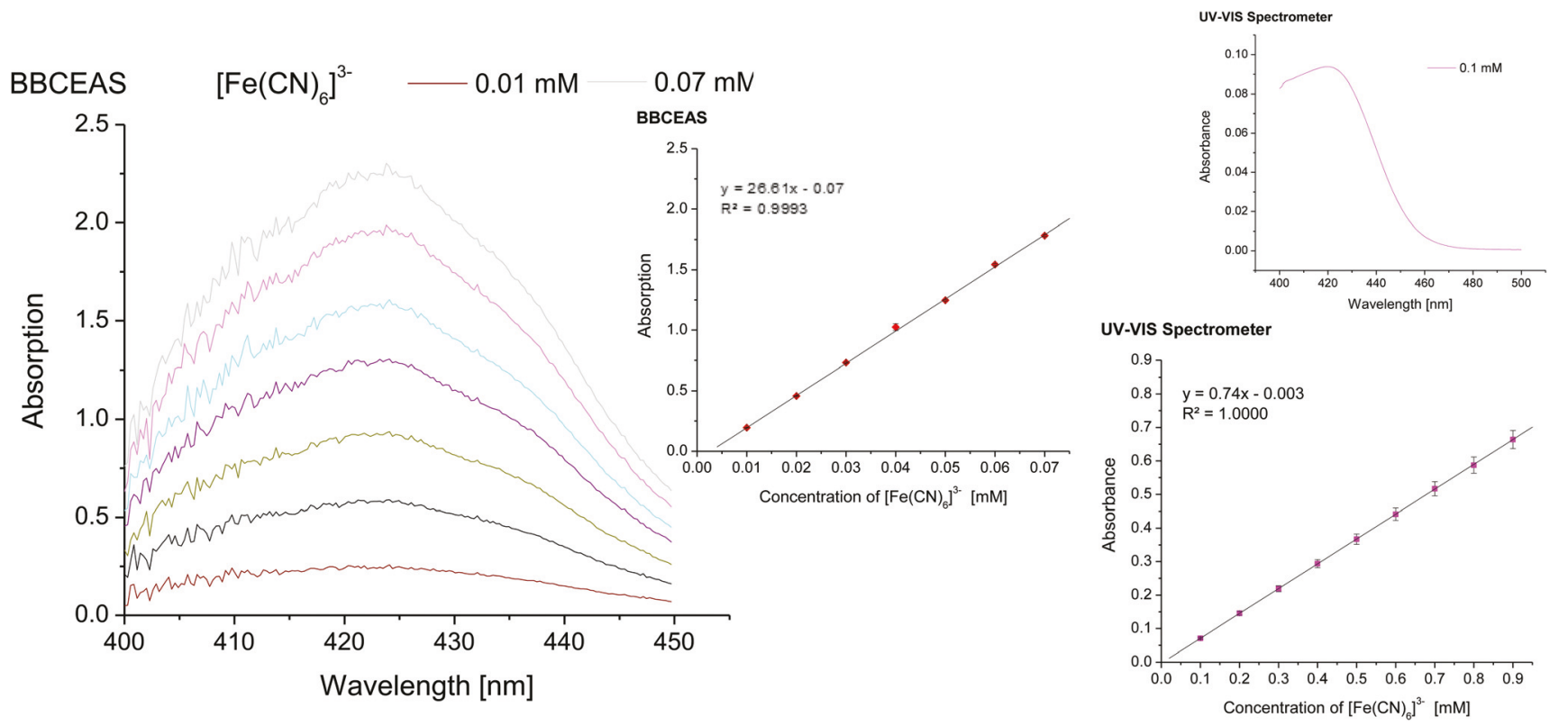

Fig. 2 Cavity enhanced absorption spectrum of ferricyanide. The inset shows the corresponding BBCEAS absorption versus concentration plot at $434 \mathrm{~nm}$ and the single pass calibration curve as well as an absorbance spectrum of ferricyanide.

Table 1 Summary of the results in terms of the base path length, the wavelength of the measurement, the CEF value, the $\alpha_{\mathrm{min}}$, the LOD of the analyte and $\triangle \mathrm{ABS}_{\min }(t)$

\begin{tabular}{llllllll}
\hline Technique & Cell & $l / \mathrm{cm}$ & $\lambda / \mathrm{nm}$ & {$\left[\mathrm{Fe}(\mathrm{CN})_{6}\right]^{3-} / \mathrm{M}$} & $\mathrm{CEF}$ & $\Delta \mathrm{ABS}_{\min }(t)$ & $\alpha_{\min }(t) / \mathrm{cm}^{-1} \mathrm{~Hz}$ \\
\hline CE stopped-flow & Flow-cell & 0.2 & 434 & $10^{-5}$ & 78 & $9.18 \times 10^{-5}$ & $1.35 \times 10^{-5}$ \\
Jasco V-630 & Flow-cell & 0.2 & 434 & $10^{-4}$ & 1 & $6.81 \times 10^{-5}$ & $7.84 \times 10^{-4}$
\end{tabular}

ascorbate at $\mathrm{pH}$ 8. The concentration of ferricyanide was kept at $0.01 \mathrm{mM}$, whereas the concentration of sodium ascorbate was varied between $0.1 \mathrm{mM}$ and $4 \mathrm{mM}$. The disappearance of ferricyanide was followed at $434 \mathrm{~nm}$ and data points were recorded at $40 \mathrm{~ms}$ intervals, the shortest measurement interval which could be reliably used. The closest corresponding measurement interval on the conventional spectrometer was $50 \mathrm{~ms}$. The kinetic traces collected over 8 seconds can be seen in Fig. 3. The reaction in all cases was over after 6 seconds.

Measurement of rate constants and reaction order. Twelve independent measurements for each sodium ascorbate concentration were conducted to obtain the pseudo-first order rate constants. Fig. 4 shows a representative BBCEAS plot of the negative natural logarithm of the absorbance change as a function of time. The inset corresponds to the negative natural logarithm of absorbance versus time plot for the conventional measurement. The equation on both plots is from a linear regression through the data points. The slope of the line equals $k_{\text {obs }}$, the pseudo-first order rate constant for the reaction. The straight line through the data confirms that the rate law is first order with respect to ferricyanide. First order kinetics also provides a very good fit to the data obtained on the conventional setup. The half-life of the cavity enhanced first order reaction, $t_{1 / 2}$ was $\sim 0.9 \mathrm{~s}$ and thus 4 half-lives were recorded during the reaction. All pseudo-first order graphs were linear up to 4 half-lives.

The second order rate constant, $k_{2}$, was obtained as the gradient of a plot of the pseudo-first order rate constants, $k_{\text {obs }}$ against sodium ascorbate concentration $\left[\mathrm{HA}^{-}\right](0.1 \mathrm{mM}-4 \mathrm{mM})$ as shown in Fig. 5. A linear regression through the data suggests that the reaction is also first order with respect to sodium ascorbate concentration. The second order rate constant obtained using BBCEAS $\left(k_{2}=3.56( \pm 0.20) \times 10^{3} \mathrm{M}^{-1} \mathrm{~s}^{-1}\right)$ was very similar to that obtained using conventional spectroscopy $\left(k_{2}=3.70( \pm 0.33) \times 10^{3} \mathrm{M}^{-1} \mathrm{~s}^{-1}\right)$. The $1 \sigma$ error limits are shown in brackets.

Measurements at $\mathbf{p H}$ 9.2. The kinetics of reaction between ferricyanide and sodium ascorbate was also followed at $\mathrm{pH} 9.2$ to increase the rate of reaction. Data was recorded at $434 \mathrm{~nm}$ at $40 \mathrm{~ms}$ intervals. The concentration of ferricyanide was $0.1 \mathrm{mM}$ for the conventional UV-visible measurements (Fig. 7b) which was the lowest that could be reliably measured, whilst the cavity enhanced data could be recorded at a much lower concentration of $3 \mu \mathrm{M}$. The sodium ascorbate concentration was varied between $0.03 \mathrm{mM}$ and $0.15 \mathrm{mM}$ for the cavity enhanced kinetic traces which can be seen in Fig. 6 .

The second order rate constant, $k_{2}$, was obtained as the gradient of the plot of $k_{\text {obs }}$ against sodium ascorbate concen- 
(a)

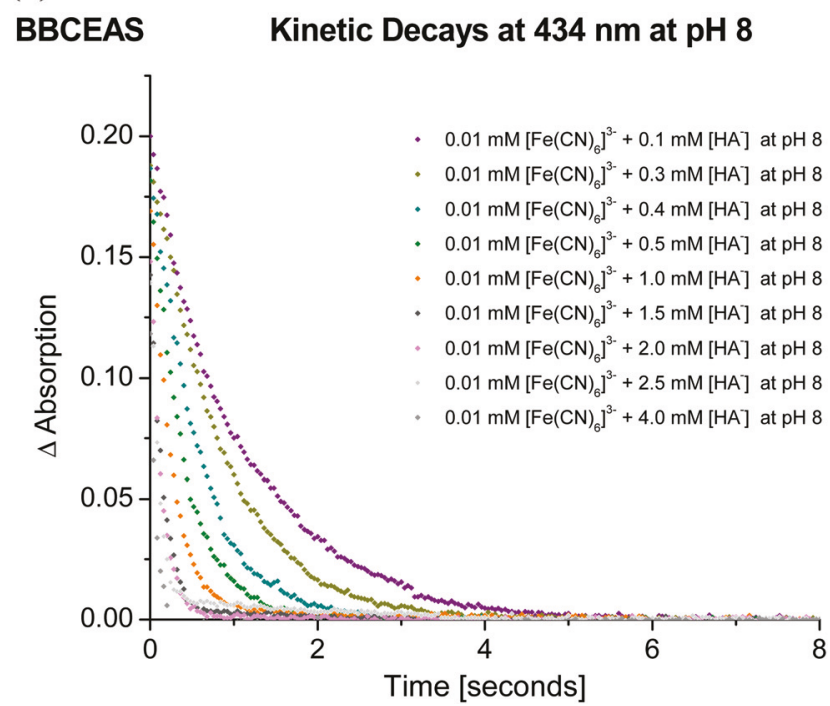

(b)

\section{UV-VIS Spectrometer Kinetic Decays at $434 \mathrm{~nm}$ at $\mathrm{pH} 8$}

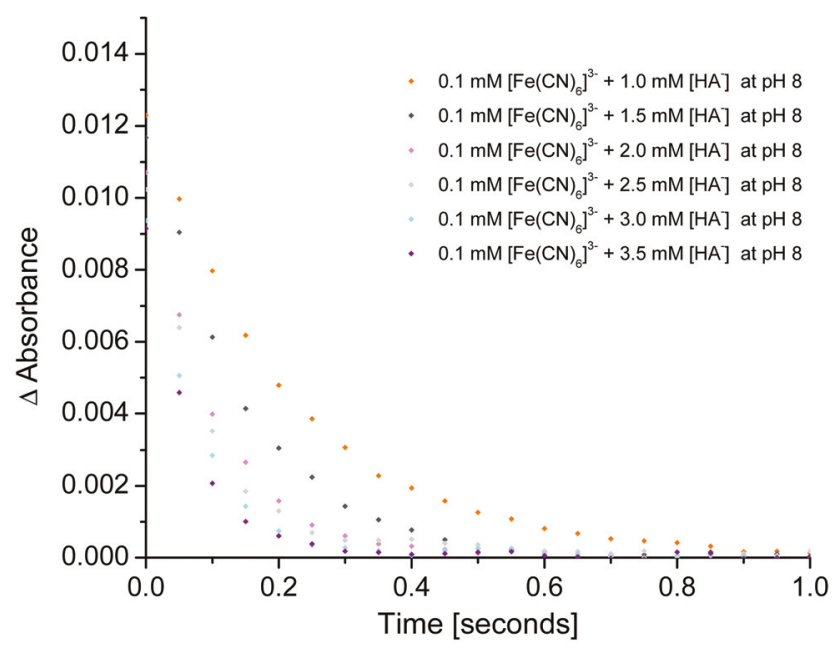

Fig. 3 (a) Cavity enhanced and (b) conventional kinetic traces collected for the reaction of $\left[\mathrm{Fe}(\mathrm{CN})_{6}\right]^{3-}$ with sodium ascorbate under pseudo-first order conditions.

(a)

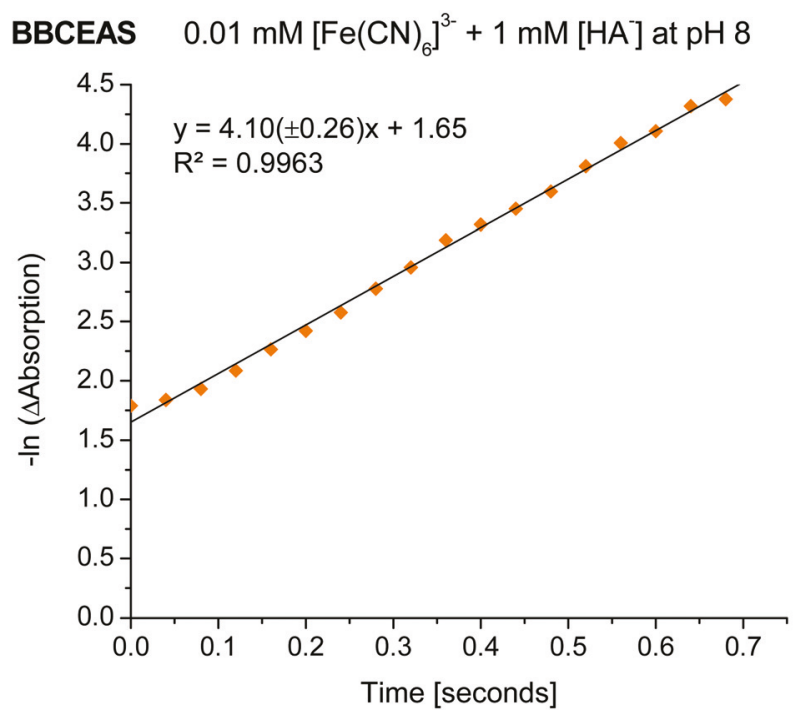

(b)

\section{UV-VIS Spectrometer $0.1 \mathrm{mM}\left[\mathrm{Fe}(\mathrm{CN})_{6}\right]^{3-}+1 \mathrm{mM}\left[\mathrm{H}_{2} \mathrm{~A}\right]$ at $\mathrm{pH} 8$}

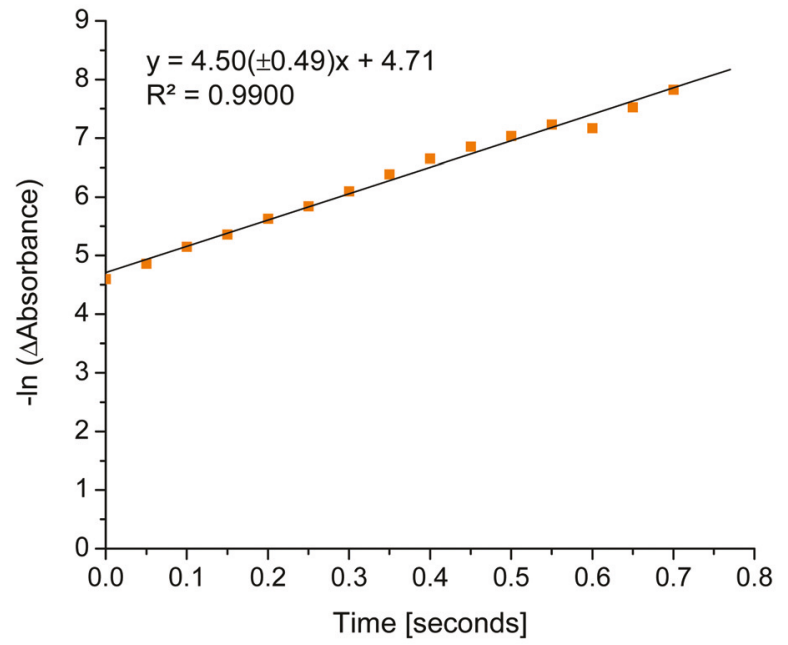

Fig. 4 Representative (a) cavity enhanced and (b) conventional pseudo-first order plots recorded for the reaction of ferricyanide with 1 mM [HA ${ }^{-}$in $\mathrm{pH} 8$ media at $25^{\circ} \mathrm{C}$. The pseudo-first order rate coefficient, $k_{\text {obs, }}$ was determined from the gradient of a linear regression through the data. The $1 \sigma$ error limits on the gradient are shown in brackets.

tration, and the results for the BBCEAS kinetic measurements at pH 9.2 is shown in Fig. 8.

\section{Discussion}

The first demonstration of BBCEAS applied to stopped-flow kinetics is reported. The BBCEAS setup was coupled to a stan- dard commercial stopped-flow accessory to follow the kinetics of reaction between ferricyanide and sodium ascorbate, at $\mathrm{pH} 8$ and $\mathrm{pH}$ 9.2. The results were compared with the kinetic data obtained using conventional UV-visible spectroscopy. The figures of merit determined include the CEF, $\alpha_{\min }$ and LOD. These values are discussed in turn. Comparison of the results is then made with previous liquid-phase cavity enhanced kinetic studies and selected short base path length liquid-phase cavity 
(a)

(b)

\section{BBCEAS $\quad \mathrm{k}_{\text {obs }}$ versus [HA] $]$ at $434 \mathrm{~nm}$ at $\mathrm{pH} 8$ UV-VIS Spectrometer $\mathrm{k}_{\text {obs }}$ versus [HA] at $434 \mathrm{~nm}$ at $\mathrm{pH} 8$}
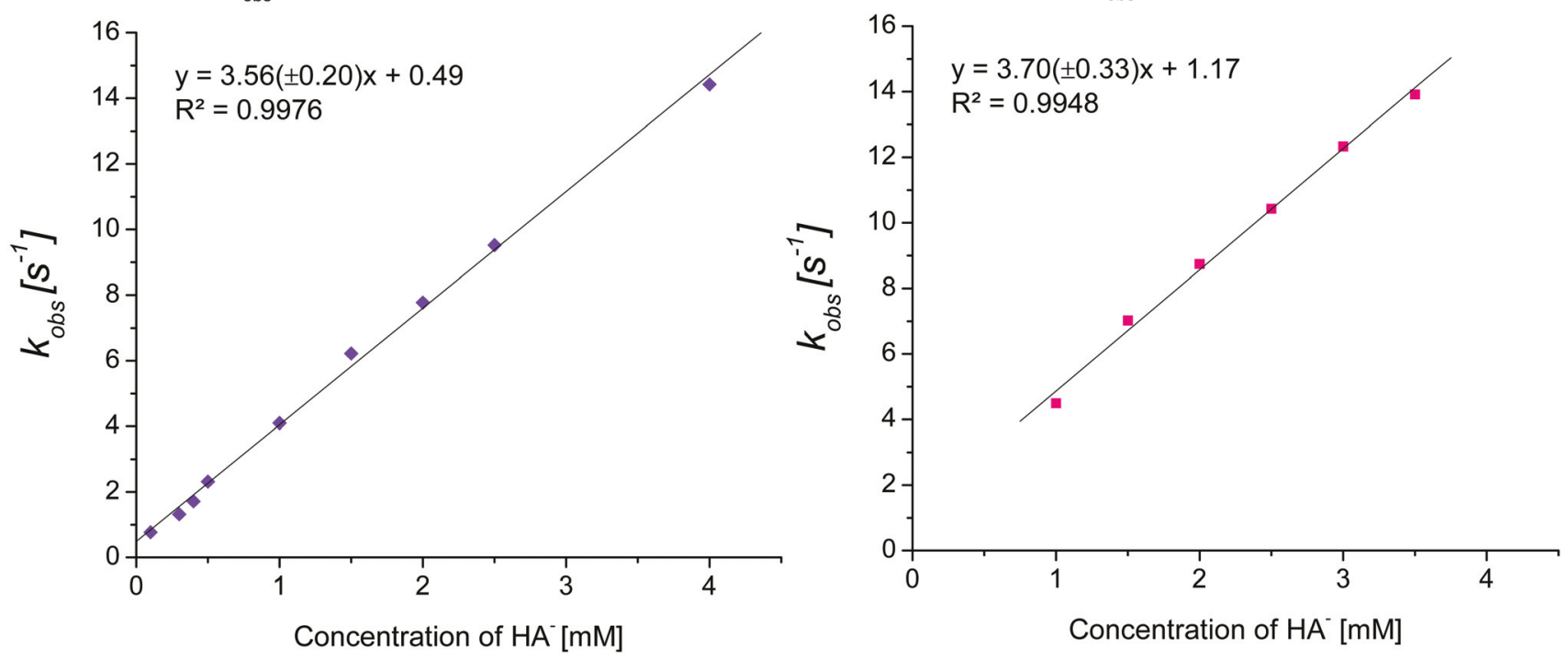

Fig. 5 Plots of the pseudo-first order rate constant $k_{\text {obs }}$ against sodium ascorbate concentration for (a) the BBCEAS setup (b) the conventional setup. The equation on both graphs allows the second order rate constants, $k_{2}$ to be determined from the gradient of the fit. The $1 \sigma$ error limits on the gradient are shown in brackets.

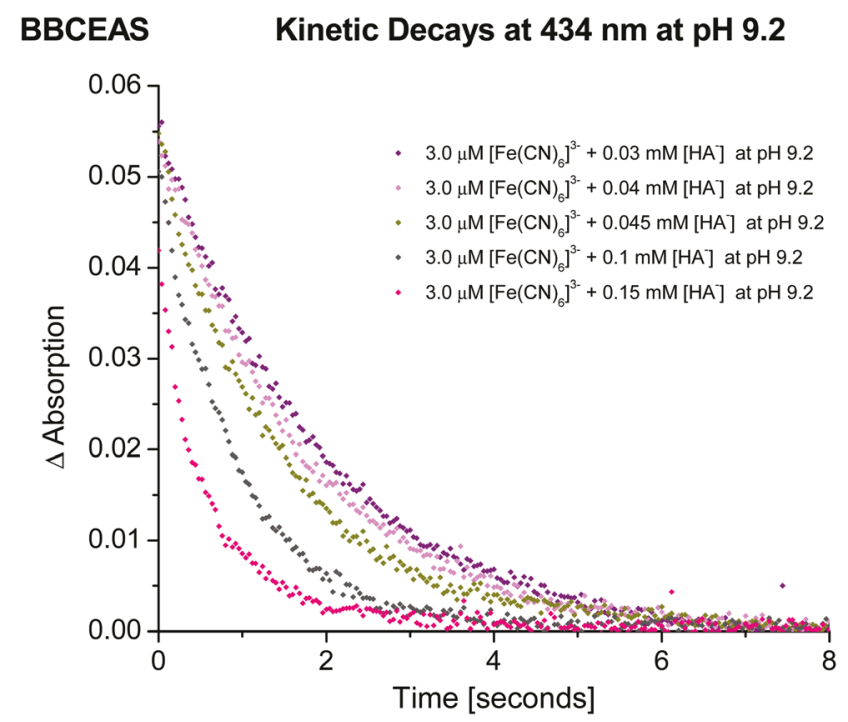

Fig. 6 Cavity enhanced kinetic traces obtained for the reaction of [Fe $\left.(\mathrm{CN})_{6}\right]^{3-}$ with sodium ascorbate under pseudo-first order conditions at $\mathrm{pH} 9.2$.

enhanced studies. Suggestions on the further improvement of the sensitivity of the experimental setup are also made.

The use of an optical cavity for measurements has resulted in a large increase in the effective path length as shown by the CEF value of 78. This forms the basis of the improvement in sensitivity achieved through the use of the cavity enhanced approach. The 58 fold increase in sensitivity meant that lower concentrations of ferricyanide and consequently sodium ascorbate could be used for the cavity enhanced measure- ments under circumstances where slower rates of reaction were needed.

At $\mathrm{pH} 8$ both the cavity enhanced and conventional measurements could be performed over approximately the same concentration range of sodium ascorbate without $k_{\text {obs }}$ becoming faster than the response time of the instrument $\left(\sim 25 \mathrm{~s}^{-1}\right)$ and produced similar $k_{2}$ values (Fig. $\left.3-5\right)$. As the cavity enhanced measurements were made with tenfold lower concentrations of ferricyanide ( $0.01 \mathrm{mM}$ versus $0.1 \mathrm{mM}$ ) measurements using up to tenfold lower concentrations of sodium ascorbate were also possible which produced slower $k_{\text {obs }}$ values but in this case this was not advantageous as the $k_{\text {obs }}$ values measured with the conventional instrument were within the response time of the instrument.

As the reaction rate increases with $\mathrm{pH}$, the measurements at $\mathrm{pH} 9.2$ were expected to be faster. For the conventional measurements the lowest concentration of ferricyanide which could be reliably measured was $0.1 \mathrm{mM}$. This restricted the minimum concentration of sodium ascorbate which could be used to $1 \mathrm{mM}$, to maintain pseudo-first order conditions. However, the measured value of $k_{\text {obs }}$ was now determined by the response time of the instrument of $\sim 25 \mathrm{~s}^{-1}$ and there was no increase in $k_{\text {obs }}$ with increasing sodium ascorbate concentration. This showed that the response time of the instrument was restricting the measurement of the true value of $k_{\text {obs }}$. For the cavity enhanced measurements, the greater sensitivity allowed a lower ferricyanide concentration of $3 \mu \mathrm{M}$ to be used. This allowed correspondingly lower concentrations of sodium ascorbate to also be used and led to slower values of $k_{\text {obs }}$ not limited by the instrumental response time as can be seen in Fig. 7 and 8. From the plot in Fig. 8 the second order 
BBCEAS $3.0 \mu \mathrm{M}\left[\mathrm{Fe}(\mathrm{CN})_{6}\right]^{3-}+0.03 \mathrm{mM}[\mathrm{HA}]$ at $\mathrm{pH} 9.2$

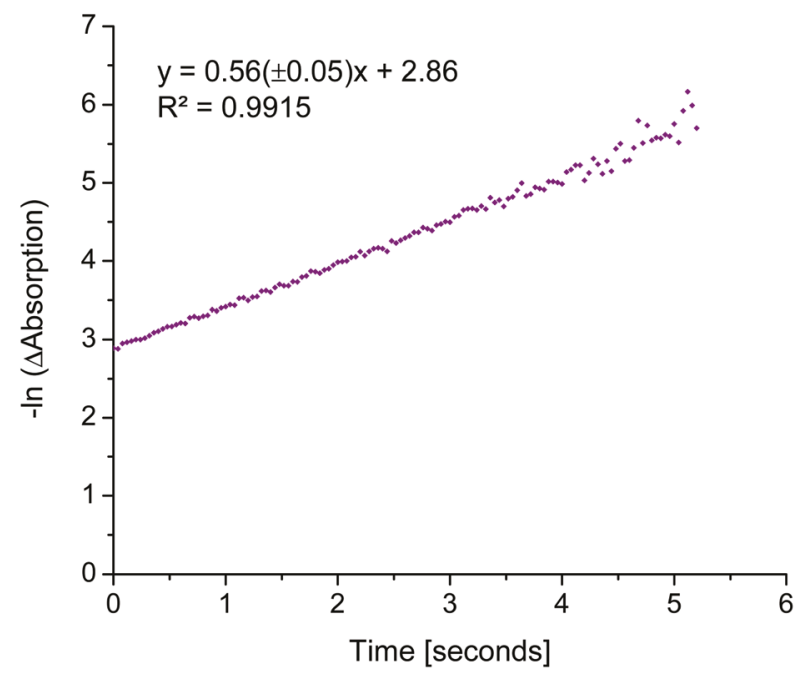

Fig. 7 Representative cavity enhanced pseudo-first order plots for the reaction of ferricyanide with $\left[\mathrm{HA}^{-}\right]$in $\mathrm{pH} 9.2$ media at $25^{\circ} \mathrm{C}$. From the equation on the plot the pseudo-first order rate coefficient, $k_{\text {obs }}$, can be determined from the gradient. The $1 \sigma$ error limits on the gradient are shown in brackets.

\section{BBCEAS $\quad \mathrm{k}_{\text {obs }}$ versus $\left[\mathrm{HA}^{-}\right]$at $434 \mathrm{~nm}$ at $\mathrm{pH} 9.2$}

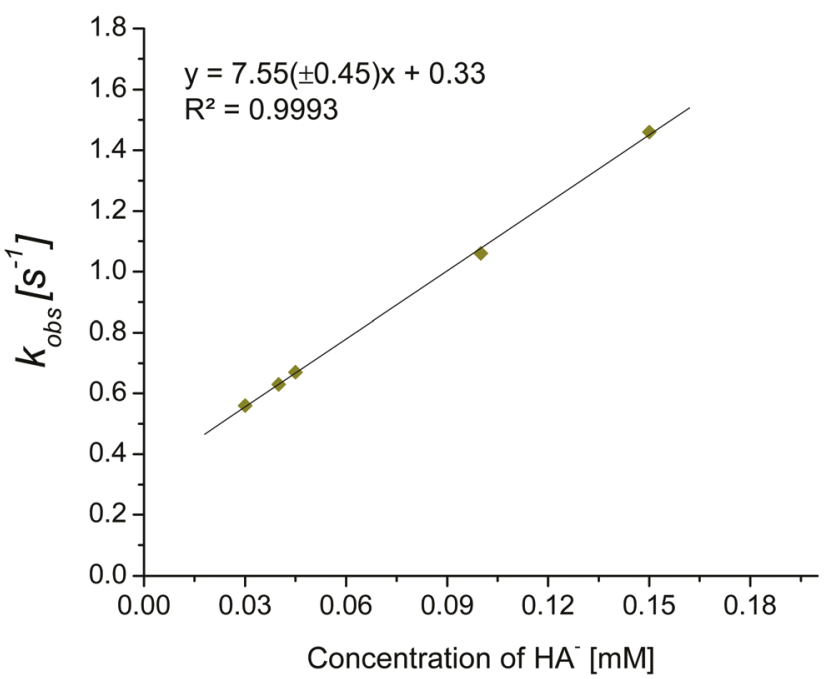

Fig. 8 A plot of the pseudo-first order rate constant $k_{\text {obs }}$ against sodium ascorbate concentration for the BBCEAS setup. The second order rate constant, $k_{2}$ can be determined from the gradient of the fit. The $1 \sigma$ error limits on the gradient are shown in brackets.

rate constant $k_{2}$ could also be determined as the gradient of the plot. These results demonstrate the value of cavity enhanced techniques in enabling the measurement of the stopped-flow kinetics of reactions which would otherwise be too fast to measure using conventional UV-visible absorption spectroscopy.
The sensitivity of the BBCEAS stopped flow apparatus was determined by the minimum detectable change in the absorption coefficient, $\alpha_{\text {min }}$. The CEF of 78 , corresponding to an effective path length of $15.6 \mathrm{~cm}$, led to a $\alpha_{\min }(t)$ value of $1.35 \times$ $10^{-5} \mathrm{~cm}^{-1} \mathrm{~Hz}^{-1 / 2}$. These figures show a $\sim 58$ fold improvement when compared to the conventional measurements $\left(\alpha_{\min }(t)=\right.$ $7.84 \times 10^{-4} \mathrm{~cm}^{-1}$ ) using a double beam UV-visible spectrometer. The main factor in the improved sensitivity of the cavity enhanced technique is due to the increased path length compared to the conventional measurement. However, the BBCEAS setup used a single beam spectrometer for measurements whilst the conventional setup used a double beam spectrometer. The advantage of a double beam spectrometer is that it is able to compensate for noise due to fluctuations in the intensity of the light source and typically leads to an improvement of $\sim 5$ fold in baseline noise and thus sensitivity. The results from this study show a much more modest improvement of $\sim 1.3$ fold in the baseline noise of the double beam spectrometer. The reasons for the less than expected improvement include, the relatively high stability and low noise of the LED light source due to the use of a low noise power supply and a large heatsink. The higher than expected baseline noise from the double beam spectrometer could be due to poor transmission of the spectrometer light source through the relatively small aperture of the flow-cell. The LED light source for the BBCEAS setup by contrast could be collimated to a relatively small beam size of about $2 \mathrm{~mm}$ in diameter and was able to pass through the flow-cell without being clipped.

Table 2 summarises the main figures of merit obtained in this study and compares these with the corresponding data available from previously reported liquid-phase kinetic studies using cavity enhanced techniques and also selected liquidphase cavity enhanced studies with short base path lengths. The comparison is made in terms of technique, the light source used, the peak wavelength of the measurement, the base path length, the lowest $\alpha_{\min }$ and the $\alpha_{\min }$ value normalised to a base path length of $1 \mathrm{~cm}$.

There have been relatively few previous cavity enhanced studies which have measured liquid-phase kinetics. Hallock et al. ${ }^{16}$ used cw-CRDS with a diode laser at $655 \mathrm{~nm}$ to follow the kinetics of the reaction between methylene blue and ascorbic acid over a timescale of a few minutes in a $23 \mathrm{~cm}$ reaction cell where the reaction solution was in direct contact with the cavity mirrors. Kiwanuka et al. ${ }^{17}$ used BBCEAS with a supercontinuum light source to study the oscillating BelousovZhabotinsky reaction over a period of six minutes in a $5.4 \mathrm{~cm}$ cuvette. Neil et al. ${ }^{18}$ used both CRDS and frequency modulated CEAS to look at the reaction between the photochemical dye thionine and 1,4-diazabicyclo[2.2.2]octane (DABCO) as a function of magnetic field in a $1 \mathrm{~mm}$ path length flow-cell at $405 \mathrm{~nm}$. They found that the frequency modulated CEAS measurements were 5.3 times more sensitive than the CRDS measurements (calculated from the reported baseline noise in absorbance units by converting to $\ln \left(\log _{e}\right)$ units and then dividing by the base path length of the cell in $\mathrm{cm}$ ). In further experiments from the same group Maeda et al. ${ }^{19}$ studied the 
Table 2 Comparison between this study and the data available from previous "short-path" liquid phase cavity studies as a function of technique, the mirror reflectivity, the light source, the cell used, the wavelength of measurement, the base path length, the lowest value of $\alpha_{\min }$, and $\alpha_{\min } \times l$

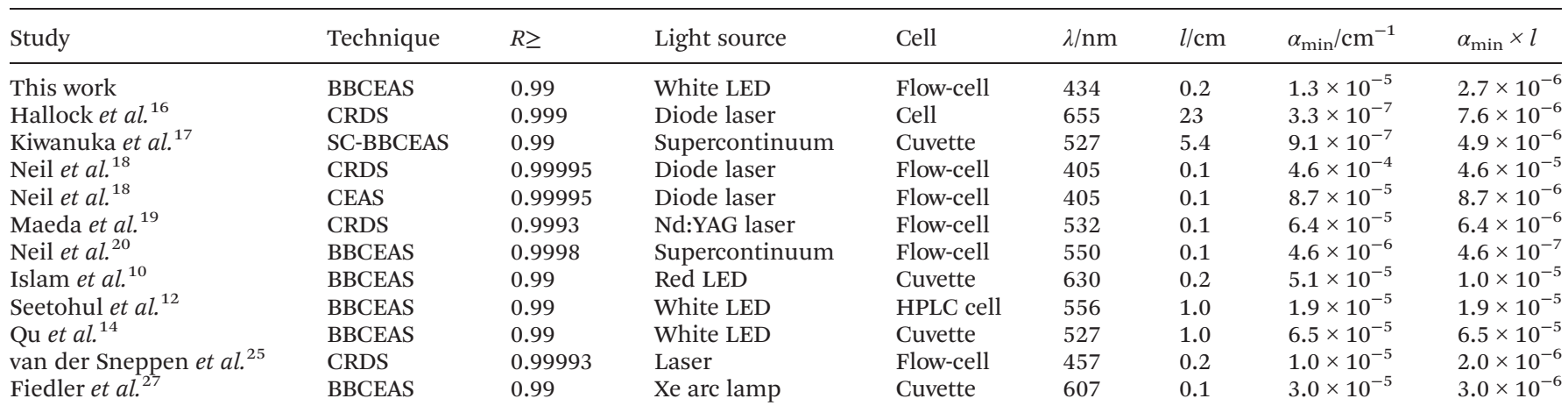

photoinduced radical pair reactions between the protein lysozyme and the photosensitizers, anthraquinone-2,6-disulphonate and flavin mononucleotide. The measurements were made using CRDS at $532 \mathrm{~nm}$ over microsecond timescales in a $1 \mathrm{~mm}$ flow-cell. Neil et l. $^{20}$ also reported further measurements with greatly improved sensitivity, using BBCEAS with a supercontinuum light source to study magnetic field effects in chemical models of a cryptochrome magnetoreceptor. When comparing the results from this study to these previous results it is important to take in account the large differences in the base path length of measurement as the $\alpha_{\min }$ value is inversely proportional to the base path length. The studies of Hallock et $a l^{16}$ and Kiwanuka et al. ${ }^{17}$ have significantly greater reported sensitivities but also used much longer base path lengths. If the $\alpha_{\min }$ values are normalised to a base path length of $1 \mathrm{~cm}$ by multiplying by the base path length in $\mathrm{cm}$ then a fairer comparison between the relative sensitivities of the different studies can be made. On this basis, our results are between 2-3 times more sensitive. Likewise, comparing with the studies of Neil et al. ${ }^{18}$ and Maeda et al. ${ }^{19}$ shows that the earlier study by Neil et al. was between 3.2 and 17 times less sensitive, whilst Maeda et al. ${ }^{19}$ was 2.4 times less sensitive. However, the later study by Neil et al. ${ }^{20}$ was 5.9 times more sensitive. Overall the relative sensitivity of this study is amongst the best whilst the complexity and cost of the experimental setup is significantly lower than the previous studies. However, the time resolution of the previous CRDS studies was significantly better than the present study as they were able to record measurements with microsecond time resolution whilst the stopped-flow setup is restricted to measurements on millisecond timescales and longer.

Comparison can also be made with previous cavity enhanced studies using short base path lengths. The most similar previous study is the BBCEAS study carried out by Islam et al., $2007^{10}$ in a $2 \mathrm{~mm}$ base path length quartz cuvette using a similar experimental setup. The improvement in sensitivity of the current study is partly due to the higher CEF value ( $\mathrm{CEF}=78$ versus $\mathrm{CEF}=51$ ) as a result of better cavity alignment and also improvements in light throughput from the cavity to the detector as a result of using a more powerful LED, better collimation of the light source and better collection of the light exiting the cavity. van der Sneppen et al., $2006^{25}$ reported HPLC-CRDS measurements in a $2 \mathrm{~mm}$ flow-cell where the cavity mirrors of the flow-cell were in direct contact with the liquid-phase analytes. The sensitivity of their measurement was similar to that of the present study, although their measurements were made using a more complex and expensive experimental setup. Fiedler et al., 2005, ${ }^{26}$ made BBCEAS measurements in a $1 \mathrm{~mm}$ cell, with $R \geq 0.99$ mirrors and a Xe arc lamp as the light source. When the base path length is taken into account the sensitivity is very similar to that obtained in this study although a more expensive high intensity short-arc Xe lamp light source was used in their study.

Other studies using a longer $1 \mathrm{~cm}$ base path length cell include that by Seetohul et al., $2009^{12}$ in a $1 \mathrm{~cm}$ path length HPLC cell coupled to a BBCEAS system. The sensitivity of their liquid-phase HPLC-BBCEAS technique, with $R \geq 0.99$ mirrors was significantly less sensitive than the current study when the base path length is taken into account. Qu et al., $2013^{14}$ also made measurements in a $1 \mathrm{~cm}$ path length cuvette using a very low cost BBCEAS setup with a webcam based detector. Their results were also considerably less sensitive than this study but used a lower cost setup.

Further improvements in the sensitivity and performance of the current experimental setup could be achieved in a number of ways. The use of a higher optical quality flow-cell would lead to a reduction in cavity losses due to scattering and reflection from the flow-cell and should produce an improvement in the CEF value and thus a higher sensitivity from a greater effective path length. It would also allow measurements to be made through the $1 \mathrm{~cm}$ path length side of the flow-cell which in principle should improve the absolute sensitivity by a factor of five. The sensitivity of the setup could also be improved by using a more powerful light source such as a supercontinuum light source and a more sensitive lower noise detector such as a thermoelectrically cooled CCD spectrometer. These changes would however greatly increase the cost of the experimental setup. The time resolution of the current cavity enhanced setup is limited to $40 \mathrm{~ms}$ as a result of the communication speed between the spectrometer and the data 
acquisition computer. Upgrading the data acquisition computer and using a spectrometer with a faster data transfer interface would allow the time resolution of the measurements to be improved to a few $\mathrm{ms}$ at which point the instrumental response time would be limited by the dead time of the stopped-flow apparatus of $\sim 10 \mathrm{~ms}$. Liquid-phase kinetic studies could also be extended into the UV region, by using suitable light sources such as UV LEDs and laser driven light sources (LDLS) ${ }^{28}$ and high reflectivity mirrors which cover the range below $400 \mathrm{~nm}$. This would increase the number of analytes which could potentially be studied, thereby broadening the applicability of the technique.

\section{Conclusion}

The first application of a cavity enhanced technique to the measurement of liquid-phase kinetics using a commercial stopped-flow kinetics accessory has been demonstrated. Comparative measurements were made on the reaction between sodium ascorbate and potassium ferricyanide at $\mathrm{pH} 8$ and pH 9.2 using a conventional UV-visible spectrometer. The BBCEAS measurements produced a CEF of 78 which led to a 58 fold improvement in sensitivity. This allowed lower concentrations of reagents to be used which proved crucial for the measurements at $\mathrm{pH}$ 9.2. At this $\mathrm{pH}$ the kinetics of the reaction was too fast to measure using the conventional spectrometer, whilst the BBCEAS measurements could be made with 30 fold lower concentration of reagent which allowed the kinetics of the reaction to be determined and demonstrated the value of cavity enhanced techniques for studying liquid-phase kinetics. Comparison with the relatively few previous cavity enhanced liquid-phase kinetic studies showed that the present measurements were amongst the most sensitive whilst using a much simpler and lower cost experimental setup. Further improvement in sensitivity could be achieved by using a higher optical quality flow-cell in conjunction with a more powerful light source and a more sensitive, lower noise detector, whilst better time resolution could be obtained by using a faster data acquisition computer and a spectrometer with a faster data transfer interface.

\section{Conflicts of interest}

There are no conflicts to declare.

\section{Acknowledgements}

Hanan Naif would like to thank the Iraqi Government for the award of PhD studentship.

\section{References}

1 G. P. Hess, Biophys. Chem., 2003, 100, 493-506.
2 G. G. Hammes, Thermodynamics and Kinetics for the Biological Sciences, New York, 2000.

3 R. Bleul, M. Ritzi-Lehnert, J. Höth, N. Scharpfenecker, I. Frese, D. Düchs, S. Brunklaus, T. E. Hansen-Hagge, F. Meyer-Almes and K. S. Drese, Anal. Bioanal. Chem., 2011, 399, 1117-1125.

4 A. N. Anthemidis, D. G. Themelis and J. A. Stratis, Anal. Chim. Acta, 2000, 412, 161-167, DOI: 10.1016/S0003-2670 (00)00751-0.

5 M. Alexovič, M. Wieczorek, J. Kozak, P. Kościelniak, I. S. Balogh and V. Andruch, Talanta, 2015, 133, 127-133, DOI: 10.1016/j.talanta.2014.04.095.

6 A. O'Keefe and D. A. G. Deacon, Rev. Sci. Instrum., 1988, 59, 2544-2551.

7 R. Engeln, G. Berden, R. Peeters and G. Meijer, Rev. Sci. Instrum., 1998, 69, 3763-3769.

8 A. O'Keefe, Chem. Phys. Lett., 1998, 293, 331-336, DOI: 10.1016/S0009-2614(98)00785-4.

9 S. M. Ball, J. M. Langridge and R. L. Jones, Chem. Phys. Lett., 2004, 398, 68-74.

10 M. Islam, L. N. Seetohul and Z. Ali, Appl. Spectrosc., 2007, 61, 649-658.

11 L. N. Seetohul, Z. Ali and M. Islam, Analyst, 2009, 134, 1887-1895.

12 L. N. Seetohul, Z. Ali and M. Islam, Anal. Chem., 2009, 81, 4106-4112.

13 S. R. T. Neil, C. M. Rushworth, C. Vallance and S. R. MacKenzie, Lab Chip, 2011, 11, 3953-3955.

14 Z. Qu, J. Engstrom, D. Wong, M. Islam and C. F. Kaminski, Analyst, 2013, 138, 6372-6379, DOI: 10.1039/ c3an01441j.

15 Z. Bajuszova, Z. Ali, S. Scott, L. N. Seetohul and M. Islam, Anal. Chem., 2016, 88, 5264-5270, DOI: 10.1021/acs. analchem.6b00375.

16 A. J. Hallock, E. S. F. Berman and R. N. Zare, Appl. Spectrosc., 2003, 57, 571-573, DOI: 10.1366/ 000370203321666614.

17 S. Kiwanuka, T. Laurila and C. F. Kaminski, Anal. Chem., 2010, 82, 7498-7501, DOI: 10.1021/ac1012255.

18 S. R. T. Neil, K. Maeda, K. B. Henbest, M. Goez, R. Hemmens, C. R. Timmel and S. R. MacKenzie, Mol. Phys., 2010, 108, 993-1003, DOI: 10.1080/ 00268971003614368.

19 K. Maeda, S. R. T. Neil, K. B. Henbest, S. Weber, E. Schleicher, P. J. Hore, S. R. MacKenzie and C. R. Timmel, J. Am. Chem. Soc., 2011, 133, 17807-17815, DOI: $10.1021 /$ ja206783t.

20 S. R. T. Neil, J. Li, D. M. W. Sheppard, J. Storey, K. Maeda, K. B. Henbest, P. J. Hore, C. R. Timmel and S. R. MacKenzie, J. Phys. Chem. B, 2014, 118, 4177-4184, DOI: $10.1021 /$ jp500732u.

21 B. Tonomura, H. Nakatani and M. Ohnishi, Anal. Biochem., 1978, 84, 370-383.

22 M. B. Davies, Polyhedron, 1992, 11, 285-321.

23 L. van der Sneppen, G. Hancock, C. Kaminski, T. Laurila, S. R. MacKenzie, S. R. T. Neil, R. Peverall, G. A. D. Ritchie, 
M. Schnippering and P. R. Unwin, Analyst, 2010, 135, 133139.

24 M. Mazurenka, A. J. Orr-Ewing, R. Peverall and G. A. D. Ritchie, Annu. Rep. Prog. Chem., Sect. C: Phys. Chem., 2005, 101, 100-142.

25 L. van der Sneppen, A. Wiskerke, F. Ariese, C. Gooijer and W. Ubachs, Anal. Chim. Acta, 2006, 558, 2-6.
26 S. E. Fiedler, A. Hese and A. A. Ruth, Rev. Sci. Instrum., 2005, 76, 023107.

27 S. E. Fiedler, A. Hese and A. A. Ruth, Chem. Phys. Lett., 2003, 371, 284-294.

28 M. Islam, L. Ciaffoni, G. Hancock and G. A. D. Ritchie, Analyst, 2013, 138, 4741-4745, DOI: 10.1039/ c3an01020a. 